\title{
Hole spin injection from a GaMnAs layer into GaAs-AlAs- InGaAs resonant tunneling diodes
}

\author{
D.H. Rodrigues ${ }^{1}$, M. J. S. P. Brasil ${ }^{2}$, M. Orlita ${ }^{3,4}$, J. Kunc ${ }^{4}$, H. V. A. Galeti ${ }^{5}$, M. \\ Henini $^{6}$, D. Taylor ${ }^{6}, Y$. Galvão Gobato ${ }^{1}$ \\ ${ }^{1}$ Universidade Federal de São Carlos, Departamento de Física, 13560-905, São \\ Carlos, SP, Brazil \\ ${ }^{2}$ Instituto de Física "Gleb Wataghin", Universidade Estadual de Campinas, 13083-970, \\ Campinas, SP, Brazil \\ ${ }^{3}$ Laboratoire National des Champs Magnétiques Intenses, CNRS-UJF-UPS-INSA, 25, \\ avenue des Martyrs, 38042 Grenoble, France \\ ${ }^{4}$ Institute of Physics, Charles University, Ke Karlovu 5, 12116 Praha 2, Czech Republic \\ ${ }^{5}$ Departamento de Engenharia Elétrica, Universidade Federal de São Carlos 13560- \\ 905, São Carlos, SP, Brazil \\ ${ }^{6}$ School of Physics and Astronomy, Nottingham Nanotechnology and Nanoscience \\ Centre, University of Nottingham, Nottingham NG7 2RD, UK
}

We have investigated the polarization-resolved electroluminescence (EL) of a pi-n GaAs/AIAs/InGaAs resonant tunneling diode (RTD) containing a GaMnAs $(x=5 \%)$ spin injector under high magnetic fields. We demonstrate that under hole resonant tunneling condition, the GaMnAs contact acts as an efficient spinpolarized source for holes tunneling through the device. Polarization degrees up to $80 \%$ were observed in the device around the hole resonance at $2 \mathrm{~K}$ under 15T. Our results could be valuable for improving the hole-spin injection in GaMnAs-based spintronic devices. 
The dilute ferromagnetic semiconductor alloy $\mathrm{Ga}_{1-x} \mathrm{Mn}{ }_{x} \mathrm{As}$ has been intensely investigated in the last years for possible spintronics applications [1-8]. Several works were focused on spin light emitting diodes (Spin-LEDs) which use GaMnAs layers as spin injectors [1-2,8]. The operation of these devices is usually based on the injection of spin polarized holes that are strongly coupled to the magnetic $\mathrm{Mn}$ ions. Since hole spin relaxation times are much shorter than electron spin relaxation times, the improvement of spin injection efficiency in those structures is a difficult issue. In the last years, it was also shown that spin-polarization of holes in p-i-p Resonant Tunneling Diodes (RTDs) can be voltage-selected, which makes such device attractive for spintronic applications [9-12]. A possible improvement of spin-LEDs could thus be obtained by using the resonant tunneling effect in association of a layer of GaMnAs acting as a spin injector in p-i-n RTDs. Under hole resonant tunneling condition, spinpolarized holes from GaMnAs layer must be efficiently injected into QW holelevels which must increase the spin polarization degree of the device.

In this work, we have investigated the polarization-resolved electroluminescence (EL) from two devices: a magnetic p-i-n GaMnAs/ GaAs/AIAs/InGaAs RTD and a reference non-magnetic structure where the GaMnAs layer is replaced by a p-doped GaAs layer. Optical measurements were performed as a function of the applied voltage under high magnetic fields up to $15 \mathrm{~T}$ parallel to the tunnel current. Under hole resonant tunneling, we have observed an enhancement of the hole-spin injection for the magnetic RTD as compared to the reference device. Polarization degrees up to $80 \%$ were observed for the GaMnAs device near hole resonant condition under $15 \mathrm{~T}$. This 
result can be attributed to an efficient hole-spin injection from the GaMnAs layer under resonant tunneling condition.

Our devices were fabricated on $n+(001)$ GaAs substrates in a Veeco Modular Genll molecular beam epitaxy (MBE) reactor equipped with a real-time wafer temperature sensor, which allows accurate monitoring of the substrate temperature during growth. The structures were grown using $\mathrm{As}_{4}$ species on Infree sample holders and consist of: (i) a Si-doped GaAs buffer layer [500nm of $\mathrm{n}$-GaAs $\left(10^{18} \mathrm{~cm}^{-3}\right), 50 \mathrm{~nm}$ of $\mathrm{n}$-GaAs $\left(10^{17} \mathrm{~cm}^{-3}\right)$, 50nm of $\mathrm{n}$-GaAs $\left(10^{16} \mathrm{~cm}^{-3}\right)$ ]; (ii) GaAs $(20 \mathrm{~nm}) / \quad \ln _{11} \mathrm{Ga}_{89}$ As $(10 \mathrm{~nm}) / \mathrm{GaAs}(20 \mathrm{~nm})$ pre-well; AIAs (5.1nm)/GaAs (4.2nm)/ AIAs (5.1nm) QW; (iii) 20nm GaAs spacer; (iv) GaMnAs (5\% Mn) ( labeled S1). A control sample (labeled S0) was also grown by replacing the GaMnAs layer with a p-type C-doped GaAs layer $\left(2 \times 10^{19} \mathrm{~cm}^{-3}\right)$. The GaAs buffer layer was grown at $550 \circ \mathrm{C}$ and the rest of the structure was grown at $450 \circ \mathrm{C}$ except for the GaMnAs which was deposited at $210 \circ \mathrm{C}$.For these experiments the layers were processed into $400 \mu \mathrm{m}$ (S0) and $200 \mu \mathrm{m}$ (S1) diameter mesas with annular $\mathrm{Ti}(10 \mathrm{~nm}) / \mathrm{Au}(100 \mathrm{~nm})$ non-annealed top contacts to allow optical access. Ohmic contacts to the back of the substrate consisted of In:Ge (30nm)/Au (100nm) annealed at $150{ }^{\circ} \mathrm{C}$ for 30 s.

Electroluminescence (EL) measurements were performed using a $0.5 \mathrm{~m}$ Andor spectrometer coupled to a Si CCD. Optical and transport measurements were performed at $2 \mathrm{~K}$ under magnetic fields up to $15 \mathrm{~T}$ parallel to the growth axis (Faraday configuration). We remark that the easy axis of the (Ga,Mn)As magnetization is in the plane of the sample (perpendicular to the growth axis). 
Right $\left(\sigma^{+}\right)$and left $\left(\sigma^{-}\right)$circularly-polarized electroluminescence spectra were selected with appropriate optical components.

Figure 1 (a) and (b) show the layer composition and a schematic diagram of our device. An InGaAs QW was added next to the n-type contact in order to analyze the spin polarization of the holes that tunnel through the RTD. Under low applied voltages, the devices show a negligible current as expected for $\mathrm{p}$-i-n RTD structures [8]. However, as the forward voltage is increased and exceed the flat-band condition voltage $\left(\mathrm{V}_{\mathrm{FB}}\right)$, holes from the $\mathrm{p}$-type contact and electrons from n-type contact can tunnel through the GaAs QW. At resonant tunneling condition, the I(V) characteristics curves can show well defined resonant peaks due to the resonant tunneling of carriers through the confined states in the conduction (E1) and valence ( $\mathrm{HH} 1$ and LH1) bands [8]. For V> $V_{\mathrm{FB}}$, the EL signal can also be observed from the GaAs and the InGaAs QWs, as illustrated in Figure 1(b).

Figure 1(c) shows typical I-V curves under zero magnetic field for S0 and S1. For both devices the I-V curves show a dominant electron resonance (labeled E1). At low voltage, the I-V curves show a quasi-linear behavior which evidences and important series resistance. The current is composed of two contributions: electrons and holes tunneling through the diode. However, we only observe one resonant peak attributed to the electron resonant tunneling for both RTDs. Hole resonances are expected to be observed in the I-V at lower voltages ${ }^{8}$ but the high contact resistance probably prevents the observation of the hole resonances from our devices. Furthermore, the potential step created by the GaMnAs layer in the valence band profile (Figure 1) should inhibit the hole-tunneling from GaMnAs and therefore the I-V of the magnetic RTD should 
be dominated by tunneling electrons [8]. This effect should reduce considerably the hole resonant peaks in the I-V characteristics of this sample making them much more difficult to be observed. However as we will show in the following, hole resonances can still be observed under high magnetic fields even though they are more pronounced on the reference sample.

Figure 2 shows typical polarization-resolved EL spectra of the GaAs and InGaAs QWs from the RTDs subjected to a $1.82 \mathrm{~V}$ bias under $15 \mathrm{~T}$. The EL peak from the GaAs QWs is observed around $1.70 \mathrm{eV}$ for both the $\mathrm{S} 0$ and the S1 structures. However, the emission from the InGaAs QW presents peaks at around $1.44 \mathrm{eV}$ for S0 and $1.41 \mathrm{eV}$ for $\mathrm{S} 1$ samples. This energy difference can be ascribed to small differences of the InGaAs QW parameters such as the QW width or the In concentration in these samples. We also remark that the GaAs QWs bands have significantly larger linewidths than the InGaAs QWs. This indicates that a significant charge build-up must occur in the GaAs QWs [13] of the biased RTDs. The excitonic spin-splitting of the QW emission bands from the So structure are almost zero $(\sim 1 \mathrm{meV}$ for the GaAs and $\sim 0 \mathrm{meV}$ for the InGaAs QW) in agreement with previous results [9]. However, we observe a small increase of the spin-splitting energies for the S1 structure for both QWs ( $2 \mathrm{meV}$ for the GaAs and $\sim 1 \mathrm{meV}$ for the InGaAs $\mathrm{QW}$ ) as compared to the S0 device. This increase could be due to small differences of the samples parameters as well as some residual $\mathrm{Mn}$ concentration and/or variations of the carrier densities in the QWs including the possible formation of charged excitons [13]. These factors may affect the g-factors of the structures. We point out that the spin splitting energies for both QWs and structures (not shown here) do not show any significant variation with the applied voltage. 
Furthermore, we remark that for both devices the GaAs QW emission shows a negative polarization degree while the emission from the InGaAs QWs is positively-polarized. The observation of different signs for the polarization degree of GaAs and InGaAs QWs is consistent with the opposite signs of carrier g-factors of GaAs and InGaAs QW [14]. We also point out that for sample $\mathrm{S} 1$ the higher energy band from the GaAs QW EL ( $\sigma$ - emission) shows a higher EL intensity. This inversion must be attributed to either the dominance of the g-factor from a minority carrier if the QW presents a large charge density of one of the carrier types or to a non equilibrium situation due to the injection of polarized carriers that recombine before attaining a thermal distribution.

Figure 3 shows I-V curves and the voltage dependence of the total EL intensity from the QWs in S1 and S0 RTDs under $15 \mathrm{~T}$. The LH1 resonant peaks become well defined for both RTDs under $15 \mathrm{~T}$. Additional structures were also observed around $1.7 \mathrm{~V}$ in the I-V curve for the S0 device that are attributed to the $\mathrm{HH} 1$ resonance. These structures were not observed for the $\mathrm{S} 1$ device probably due to the potential profile from this structure which tends to inhibit the hole tunneling. We also observe that for the $\mathrm{S} 1$ device the total GaAs QW EL intensity shows a strong correlation between the IV curve and both the hole and electron resonances. In contrast, in the case of the S0 device, the intensity of the GaAs QW emission is correlated solely to the electron resonant peak from the I-V curve. If we consider a simple model, the GaAs QW EL intensity should be given by the product of the electron and the hole densities that tunnel into the $\mathrm{QW}$, while the I(V) curve should be proportional to the sum of the electrons and holes tunneling through the RTD. Particularly, as we discussed before the hole tunneling is inhibited in sample S1. As a 
consequence, the holes must be always considered minority carriers in the GaAs QW of the S1 structure, decreasing to negligible density values for voltages beyond the hole resonant voltages. Therefore, the EL intensity in S1 should be very sensitive to the hole resonances. This effect is not observed for the S0 device, which indicates that the hole densities should be more similar to the electron densities for this structure. The correlation effect between the I(V) curve and the EL intensity is diminished for the InGaAs QWs which can be explained by the fact that in this case the EL intensity should mainly represent the density of holes tunneling through both AlAs barriers, whereas the second tunneling is basically a non-resonant process. For both structures, the InGaAs QW EL intensity increases with the voltage and shows a tendency of saturation for high voltages that must reflect the overall charge distribution along the RTD.

Figures $4(\mathrm{a})$ and (b) illustrate the voltage dependence of the current and the degree of circular polarization (DCP) from the EL emission of both QWs and RTD structures at $15 \mathrm{~T}$. The DCP was calculated by: $\left(I^{\sigma+}-I^{\sigma-}\right) /\left(I^{\sigma+}+I^{\sigma-}\right)$, where $I^{\sigma+}$ and $I^{\sigma-}$ are the integrated intensities of the right $(\sigma+)$ and left $\left(\sigma^{-}\right)$circularlypolarized emissions. As a general behavior for both structures, the InGaAs QWs show a positive polarization, while the GaAs QWs have a negative polarization for all applied voltages. However, the DCP from both the GaAs and the InGaAs QWs from the So structure do not present any significant voltage dependence. On the other hand, the DCP for the QWs from S1 shows clear variations with the applied voltage, particularly for the GaAs QW. In fact, considering the modulus of the DCP of the GaAs QW emission from S1, we observe a correlation between the DCP and the I(V) curve: the DCP increases considerably around the voltages corresponding to hole resonances and it also 
shows some increase just after the electron resonance, when the relative number of holes compared to electrons in the GaAs QW increases. Considering that the GaAs QW excitonic spin-splitting does not show any significant voltagedependence, the DCP variation from S1 cannot be explained by a simple thermal occupation effect. These results thus indicate that the DCP voltage variation must be related to the injection of spin polarized holes along the structure. Furthermore, as the effect is rather strong for the magnetic structure but it is not observed in the reference sample, this points out to an efficient injection of spin-polarized holes from the GaMnAs layer under resonant tunneling conditions. The spin injection effect can also be reinforced by the reduced hole densities of the $\mathrm{S} 1$ structure as compared to the reference sample, since minority carriers play a more important role defining the polarization degree from a QW emission. Figure 4(c) shows the magnetic field dependence of polarization degree from the GaAs QW emission for S1 and S0 at $2 \mathrm{~K}$ for $1.78 \mathrm{~V}$ (onset of hole tunneling). It was observed that for all magnetic fields the polarization degree is higher for the $\mathrm{S} 1$ which indicates an important contribution of spin injection from GaMnAs layer.

Concerning the InGaAs QW emission, we observe that the DCP from the S1 structure shows a bias dependence only for low voltages, around the onset of hole resonant tunneling. As mentioned before the InGaAs QW spin-splitting remains mainly constant for all applied voltages. Therefore, this low-voltage dependence of the InGaAs QW DCP should also reflect some non-thermal occupation effect. As the InGaAs QW must mainly present a constant density of electrons from the adjacent $n$-doped GaAs contact, its emission must basically reflect the dynamics of the holes that tunnel through the two barriers of the RTD 
structure. Therefore, our results indicate that the injection of spin-polarized holes from the GaMnAs layer (spin aligner) should be rather efficient under low voltages conditions when the carrier densities along the structure are relatively small, so that some hole spin-polarization persists after carrier tunneling through the second RTD barrier. Based on this interpretation, the injection of spin-polarized holes results in a decrease of the positive DCP from the InGaAs QW emission. In fact, this polarization signal is consistent with the observed increase of the modulus of the negative GaAs QW DCP at hole resonances. We also remark that for high voltages, the InGaAs QW DCP from the magnetic structure tends to a constant value, independent of the applied voltage as observed for the reference structure, and it should then represent the thermal occupation based on the excitonic g-factor of the InGaAs QW. The fact that the thermal DCP values of the InGaAs QWs from the two structures are different could be explained by different g-factors, which is indeed consistent with the distinct peak energies observed for the EL emission from the InGaAs. Non intentional variations of the composition and thickness of the InGaAs QWs could explain both the variation of the EL emission energy as well as the excitonic g-factors.

In conclusion, we have investigated the circular polarization degree of the electroluminescence emission from $p-i-n$ RTDs with a $\mathrm{Ga}_{1-x} \mathrm{Mn}_{x} \mathrm{As}(\mathrm{x}=5 \%)$ type contact and a reference device with similar design and C-doped contact under high magnetic field (15T) and Faraday configuration. We have observed a voltage-controlled polarization-degree from the QW emission solely for the GaMnAs RTDs. Polarization values up to $-80 \%$ at $2 \mathrm{~K}$ and $15 \mathrm{~T}$ were observed for the GaAs QW around hole resonant tunneling condition. We have also 
observed a voltage dependence of the polarization degree from the InGaAs QW of the magnetic RTD at the onset of hole resonance. Our results provide strong evidence that the GaMnAs contact acts as a spin-polarized source for tunneling carriers along the structure. This finding could be useful to improve the efficiency of spin injection in GaMnAs spintronic devices.

The financial supports from the Brazilian Agencies FAPESP (grant numbers 2012/24055-6 and 2014/50513-7), CAPES, CNPq, the UK Engineering and Physical Sciences Research Council and the EuroMagNET II are gratefully acknowledged. 


\section{References}

[1] Ohno Y, Young DK, Beschoten B, Matsukura F, Ohno H, Awschalom DD, 1999 Nature 402790

[2] Ohno H, 1998 Science 281951.

[3] Sanghoon Lee, Chung JH, Liu X, Furdyna JK and Kirby B J, 2009 Materials Today 1214

[4] Jungwirth T., Sinova J, Mašek J, Kučera J, and MacDonald AH 2006 Rev. Mod. Phys. 78809

[5] Mattana R, George JM, Jaffre`s H, Nguyen Van Dau F, Fert A, Lépine B, Guivarc'h A, and Jézéquel G, 2003 Phys.Rev.Lett 90166601

[6] Muneta I, Ohya S, and Tanaka M, 2012 Appl. Phys. Lett. 100, 162409

[7] Ohya S, Takata K, Tanaka M, 2011Nature Physics 7342

[8] Thomas O, Makarovsky O, Patanè A, Eaves L, Campion RP, Edmonds KW, Foxon CT, and Gallagher BL, 2007 Appl. Phys. Lett. 90082106

[9].de Carvalho HB, Galvão Gobato Y, Brasil MJSP, Lopez-Richard V, Marques GE, Camps I, Henini M, Eaves L, and Hill G, 2006 Phys. Rev. B 73, 155317

[10] de Carvalho HB, Brasil MJSP, Galvão Gobato Y, Marques GE, Galeti HVA, Henini M, and Hill G, 2007 Appl. Phys. Lett. 90062120

[11] dos Santos L F, Galvão Gobato Y, Marques GE, Brasil MJSP, Henini M, Airey R, 2007Appl. Phys. Lett. 91073520

[12] dos Santos L F, Galvão Gobato Y, Marques GE, Brasil MJSP, Henini M, Airey R, 2008 Appl. Phys. Lett. 92143505

[13] Vercik A, Galvão Gobato Y, Brasil MJSP, 2002 J. Appl. Phys. 921888

[14] Ito T, Shichi W, Morisada S, Ichida M, Gotoh H, Kamada H, Ando H, 2006 Phys. stat. sol. (c) 33496 
[15] Snelling M J, Blackwood E, McDonagh C J,. Harley R T, and Foxon CTB, 1992 Phys. Rev. B 45 R3922 


\section{Figure Captions}

Figure 1. (a) and (b) Schematic band diagram of our device under bias . (c) Current Voltage characteristics curves for 0T and 2K for the GaMnAs RTD (S1) and reference device (S0).

Figure 2. Typical polarization resolved EL spectra of GaAs and InGaAs QWs at 1.82V under $15 \mathrm{~T}$ and $2 \mathrm{~K}$ for $\mathrm{S} 0$ and $\mathrm{S} 1$ devices.

Figure 3. Total EL intensity versus applied voltage from $\mathrm{GaAs}$ and InGaAs QWs and $\mathrm{I}(\mathrm{V})$ characteristics curve under $15 \mathrm{~T}$ and $2 \mathrm{~K}$ for $\mathrm{S} 0$ and $\mathrm{S} 1$.

Figure 4. Voltage dependence of circularly polarization degree in S0 and S1 for (a) InGaAs QW emission and (b) GaAs QW emission at 15T and 2K. The voltages corresponding to heavy hole $(\mathrm{HH} 1)$, light hole $(\mathrm{LH} 1)$ and electron resonance (E1) are indicated and corresponds to the maximum polarization degree.(c) Magnetic field dependence of circularly polarization degree at $1.78 \mathrm{~V}$ for S1 and S0 at 2K. 
(a)

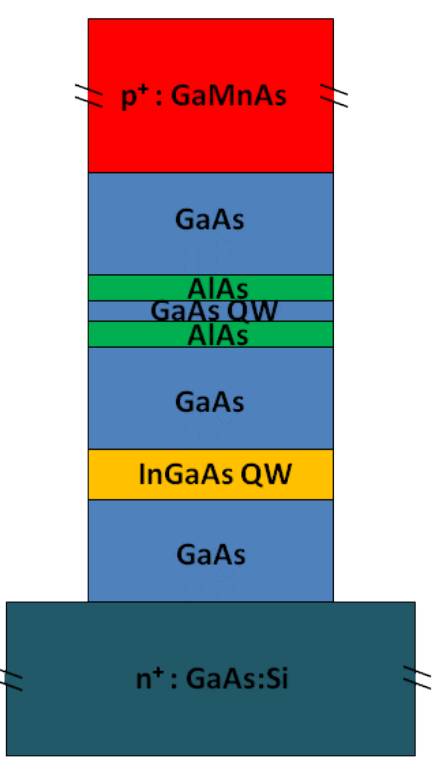

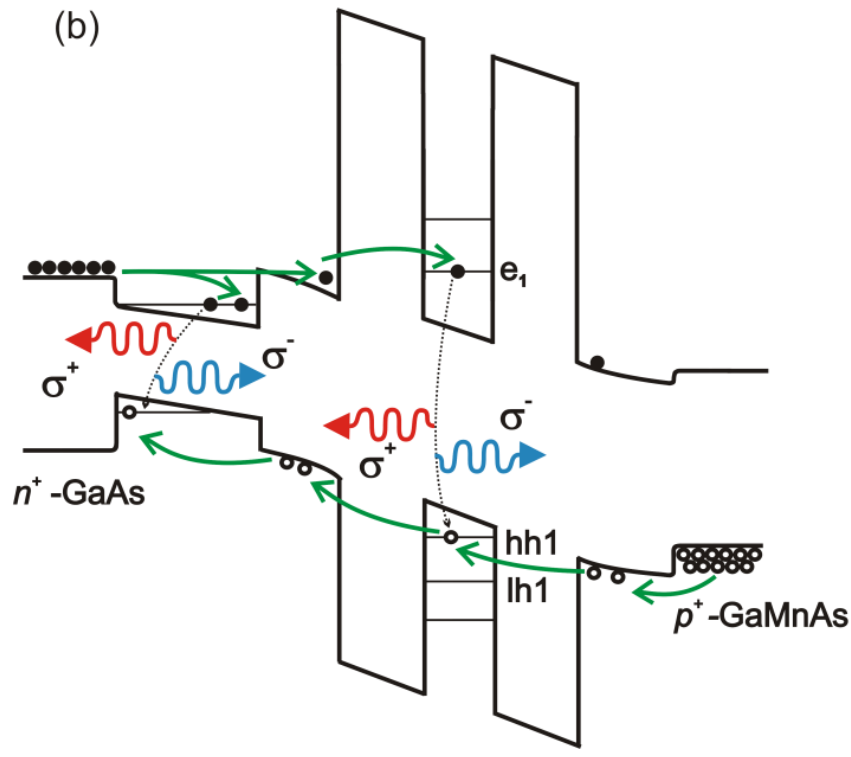

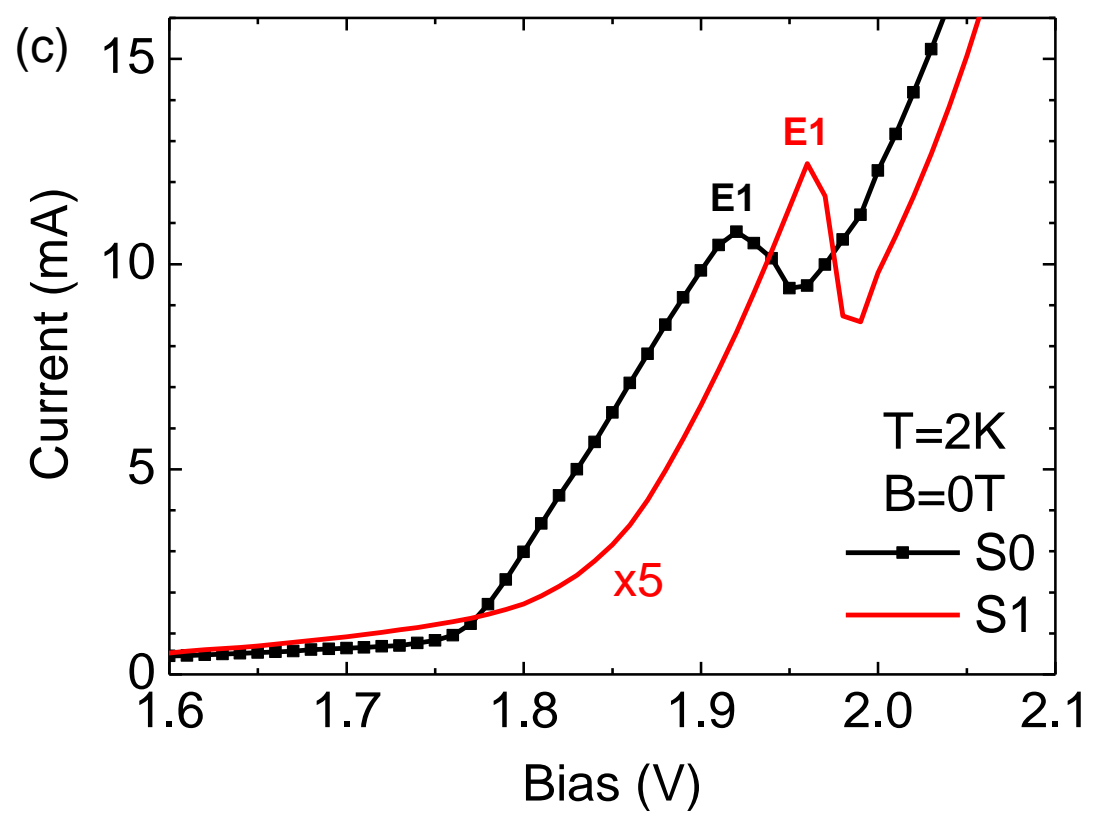

Figure 1 

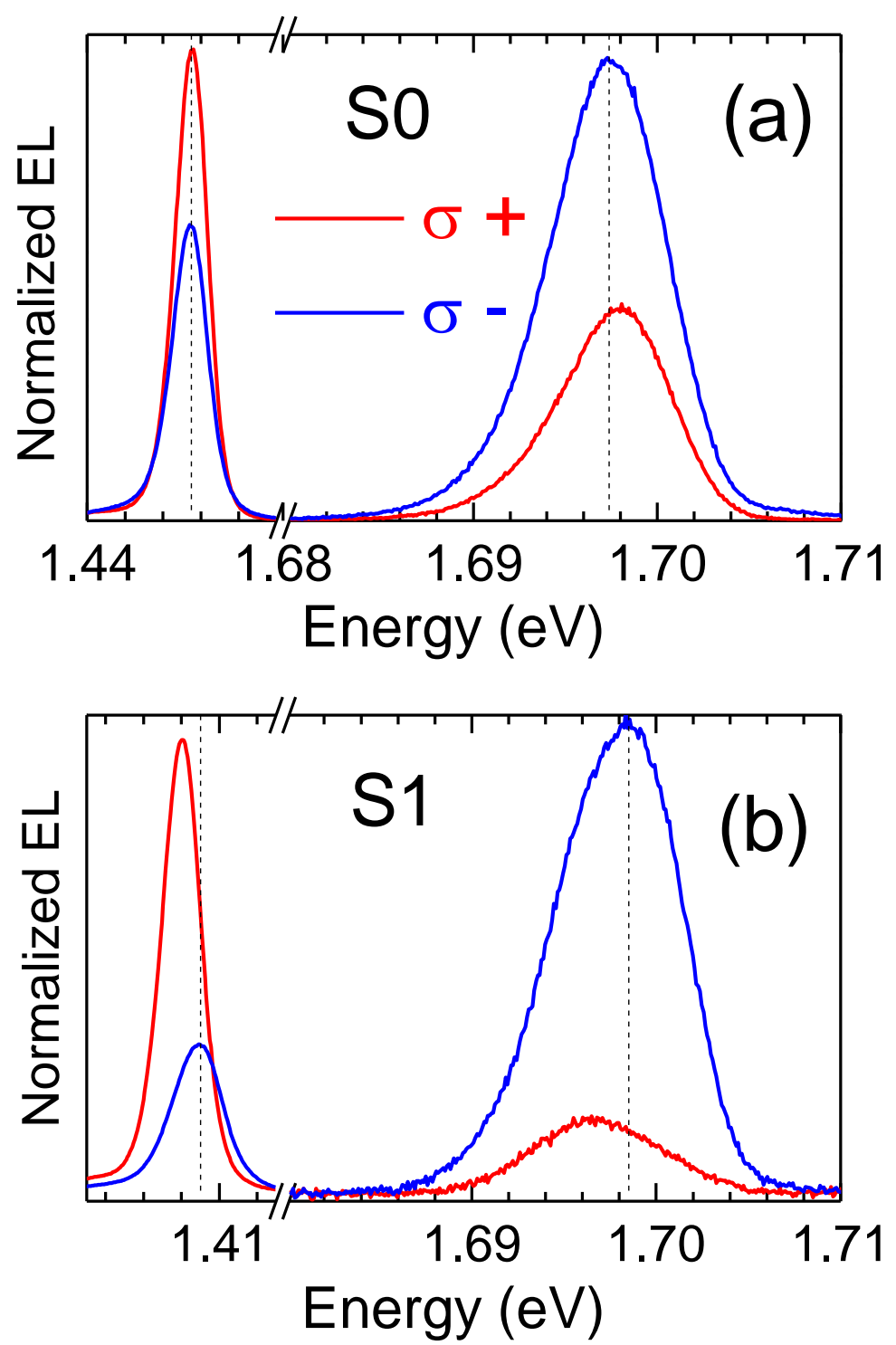

Figure 2 

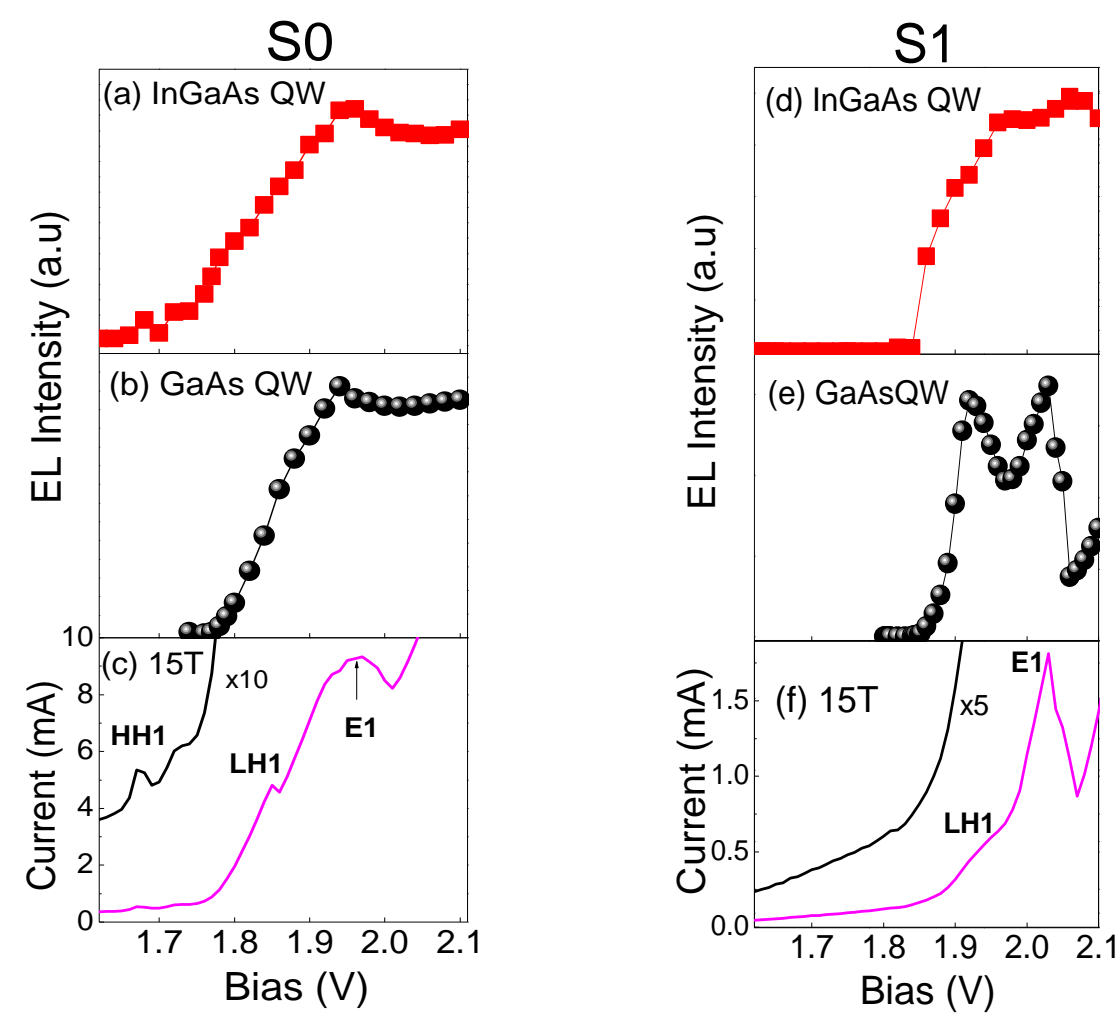

Figure 3 

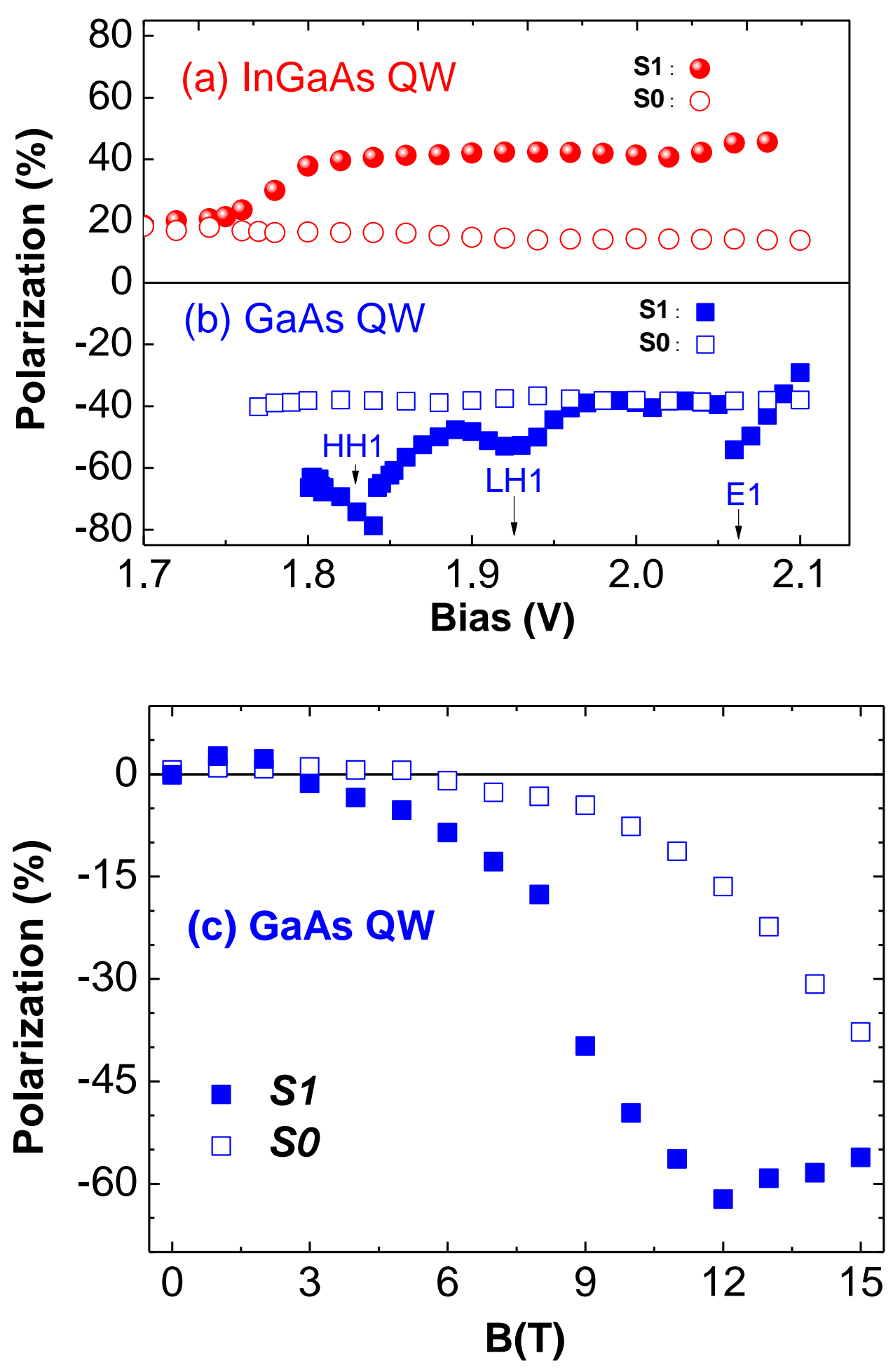

Figure 4 